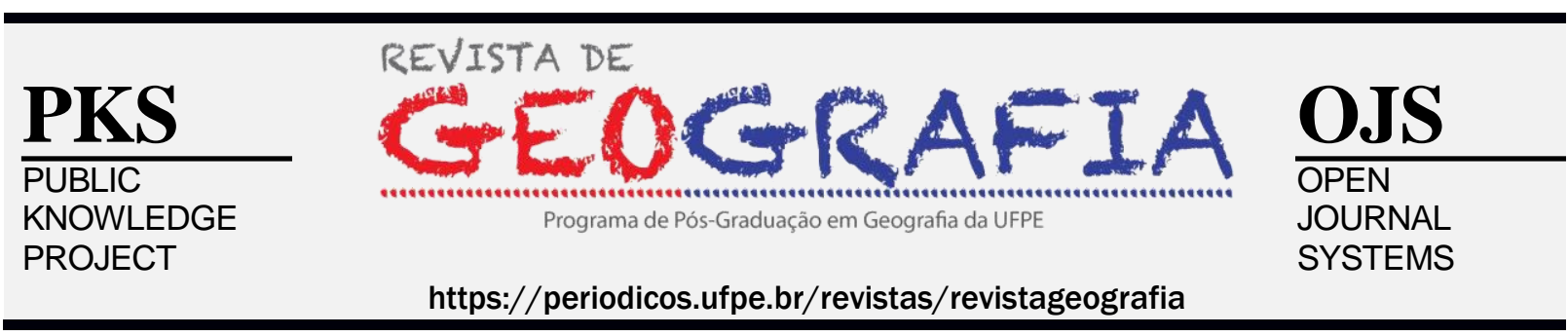

\title{
VARIABILIDADE ESPAÇO-TEMPORAL DA PRECIPITAÇÃO NA MICRORREGIÃO DE ÓBIDOS, PARÁ: UM ESTUDO UTILIZANDO A CPC MORPHING TECNIQUE (2005 - 2017)
}

\author{
Andreza Dantas Santos ${ }^{1}$, Ignázio Oliveira Bechis ${ }^{2}$, Gabriel Figueiredo Miranda ${ }^{3}$, \\ Jefferson Inayan Oliveira Souto ${ }^{4}$, Norma Ely Santos Beltrão ${ }^{5}$
}

\author{
${ }^{1}$ Instituto de Geociências (IG) - UFPA. E-mail: andrezadantast@gmail.com \\ ${ }^{2}$ Instituto de Geociências (IG) - UFPA. E-mail: iobechis@gmail.com \\ ${ }^{3}$ Instituto de Geociências (IG) - UFPA. E-mail: gabrielfm99@yahoo.com.br \\ ${ }^{4}$ Programa de Pós-Graduação em Ciências Ambientais (PPGCA) - UEPA. E-mail:inayansouto@gmail.com \\ ${ }^{5}$ Programa de Pós-Graduação em Ciências Ambientais (PPGCA) - UEPA. E-mail:normaely@uepa.br
}

Artigo recebido em 24/10/2018 e aceito em 14/06/19

\begin{abstract}
RESUMO
A precipitação desempenha um papel importante na avaliação de riscos relacionados a enchentes, secas e gestão de recursos hídricos, especialmente em regiões tropicais. O objetivo do estudo foi implementar uma análise estatística para estudar a variabilidade espacial e temporal da precipitação na microrregião de Óbidos, através de séries temporais do CPC MORPHing (CMORPH) tecnique durante o período de 2009 a 2017, assim como sua relação com o El Niño-Oscilação Sul (ENOS) através da normalização de dados. Os resultados mostram que os maiores acúmulos pluviométricos na microrregião de Óbidos estão concentrados principalmente entre os meses de dezembro a maio. Em termos de padrão espacial, ocorreram alterações significativas associadas ao fenômeno de ENOS, com déficit pluviométrico durante a ocorrência de El Niño e uma qualidade do regime de precipitação em ocorrências de La Niña em comparação com os anos neutros. Além disso, o alto desempenho dos dados do CMORPH possibilitou a identificação de diferentes padrões de circulação atmosférica, representando o ciclo anual da região.
\end{abstract}

Palavras-chave: CMORPH; El Niño; La Niña; Sensoriamento Remoto; Amazônia

\section{SPATIOTEMPORAL VARIABILITY OF PRECIPITATION IN ÓBIDOS MICRORREGION, PARÁ: A STUDY APPROACHING CPC MORPHING TECNIQUE (2005-2017)}

\begin{abstract}
Rainfall plays a very important role in assessing risks related to floods, droughts and water resource management, especially in tropical regions. The objective of the study was to implement a statistical analysis to study the spatial and temporal variability of precipitation in the Óbidos microrregion through time series of CPC MORPHing (CMORPH) tecnique during the period from 2009 to 2017, as well as it is relation with El Niño Southern regulation (ENSO) through data normalization. The results show that the greatest pluviometric accumulations in the Óbidos microregion are mainly concentrated between the months of December and May. In terms of spatial pattern, there were significant changes associated with the ENSO phenomenon, with rainfall deficit during El Niño and a quality of the precipitation regime in La Niña occurrences in comparison with the neutral years. In addition, the high performance of the CMORPH data allowed the identification of different patterns of atmospheric circulation, representing the annual cycle of the region.
\end{abstract}

Keywords: CMORPH; El Niño; La Niña; Remote Sensing; Amazon 


\section{INTRODUÇÃO}

A precipitação é uma das variáveis mais importantes para a região tropical, frequentemente usada para detectar a extensão e magnitude das mudanças climáticas e alterações no ciclo hidrológico (ZHAN et al., 2015; TOTÉ et al., 2015). A compreensão de sua variabilidade espacial e sazonal em cada região também é essencial para a agricultura e para vários setores da economia (BELAY et al., 2014), sendo necessário para o planejamento das atividades humanas e desenvolvimento local (FAO, 2015). Contudo, a irregularidade na quantidade e distribuição regional de pluviômetros, além da ocorrência frequente de lacunas e falta de homogeneidade nas séries temporais de precipitação, tornaram-se um dos fatores limitantes para seu monitoramento (TAPIADOR et al., 2012; DUAN et al., 2016).

Nesse contexto, estudos relacionados aos dados de sensoriamento remoto em regiões tropicais (LETTENMAIER et al., 2015; AWANGE et al., 2016), constataram que estes tendem a detectar e fornecer informações abundantes sobre a variabilidade da precipitação, com estimativas de alta resolução espacial e temporal (XIE; XIONG, 2011; NIKOLOPOULOS et al., 2013; MEI et al., 2014), em áreas onde os dados de precipitação são escassos ou ausentes. Desta forma, os algoritmos para estimar dados de precipitação através de observações por satélite estão cada vez mais aprimorados e em constante aperfeiçoamento (LIU et al., 2015), à medida que surgem novas técnicas e correções para as informações finais, onde cita-se, a exemplo, os estudos desenvolvidos por Schneider et al. (2014), Balsamo et al. (2015), Funk et al. (2015), Ashouri et al. (2015), Wen et al. (2016) e Sun et al. (2018).

Com essa perspectiva, as diversas informações obtidas por sensoriamento remoto, possibilitam compreender as alterações no balanço hídrico e clima regional no Brasil (KAYANO et al., 2013; MARENGO et al., 2013; TORRALBA et al., 2015), que possui uma forte influência da variabilidade interanual da Temperatura da Superfície do Mar (TSM) nas áreas dos oceanos Pacífico Equatorial e Atlântico Tropical (SOUZA et al., 2018), associada ao El Niño Oscilação Sul e o Gradiente Inter-hemisférico (SATYAMURTY et al., 2013a, 2013b), respectivamente. Estes eventos afetam ao longo do ano, o ciclo da precipitação e vazões das bacias hidrográficas, ocasionado grandes estiagens e cheias em diferentes regiões (MARENGO; ESPINOZA, 2015; JIMÉNEZ-MUÑOZ et al., 2016; TYAQUIÇÃ et al., 2017).

Adicionalmente, tendo em vista o potencial das técnicas que envolvem sensoriamento remoto, os dados do CPC MORPHing tecnique foram utilizadas com a finalidade de regionalizar os padrões de precipitação anuais no período de 2005 a 2017, bem 
como, associar os mecanismos de precipitação sobre a microrregião de Óbidos, gerando informações úteis para o planejamento de atividades locais.

\section{MATERIAL E MÉTODOS}

\section{Área de estudo}

O estudo abrangeu a microrregião de Óbidos, localizada no Baixo Amazonas, Estado do Pará (Figura 1). A microrregião é formada por cinco municípios: Oriximiná; Faro; Óbidos; Juruti e Terra Santa. Estes municípios compreendem uma área territorial de aproximadamente $157.600 \mathrm{~km}^{2}$, com uma população estimada de 184.294 habitantes (IBGE, 2010).

Apesar da região microrregião fazer parte do bioma Floresta Tropical, o clima na região é muito mais seco que o das demais áreas a leste e a oeste do Baixo Amazonas (KOSUTH et al., 2009). O clima é classificado como Tropical de Monções (Köppen Amw), com duas estações distintas: o período chuvoso, conhecido localmente como inverno, e o período seco, conhecido localmente como verão (JUNK; PIEDADE, 2005).

Figura 1. Localização geográfica da microrregião de Óbidos, Estado do Pará.

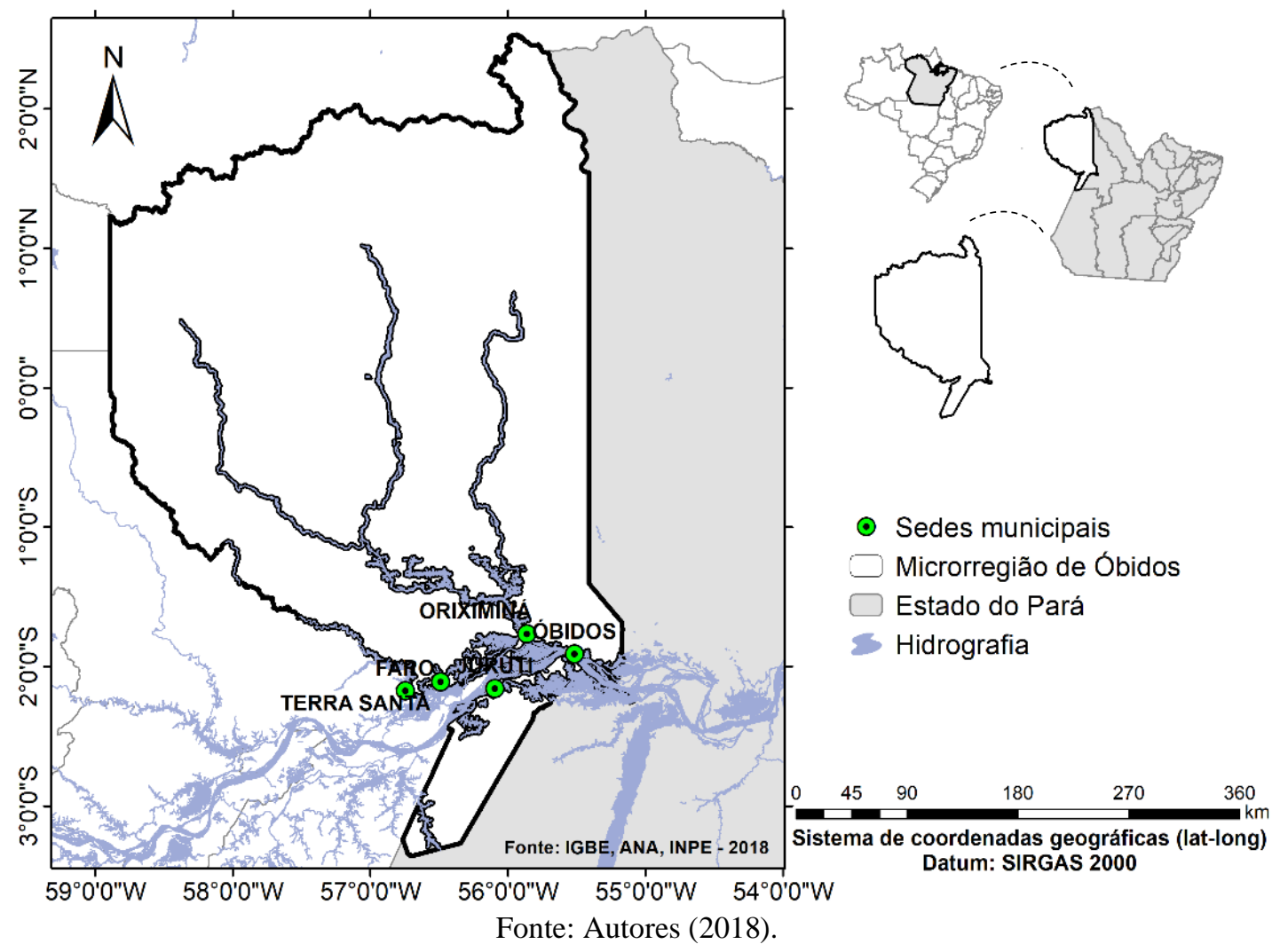

Dados de precipitação 
Revista de Geografia (Recife) V. 36, No. 2, 2019 - ISSN: 2238-6211

Neste trabalho, optou-se por utilizar as estimativas de precipitação do CPC CMORPHing (CMORPH) tecnique (JOYCE et al., 2004; JOYCE; XIE, 2017) do National Oceanic and Atmospheric Administration (NOAA). Trata-se de um conjunto de dados estimados que produzem análises globais de alta resolução espaço-temporal através de imagens dos sensores de infravermelho e micro-ondas passiva, usando a interpolação de Lagrange (GUO et al., 2015; BECK et al., 2017).

Das três versões do produto, o CMORPH V1.0 destaca-se pela grade de 0,0727 x $0,0727^{\circ}$ (aproximadamente $8 \mathrm{~km}$ ) e escala horária de 30 minutos. Apresenta alta acurácia quantitativa, comprovado em diversos estudos para a Amazônia (FALCK et al., 2016; ROSA et al., 2016; SANTOS et al., 2017; GERMANO et al., 2017). Os dados foram agregados para uma escala anual no período de junho de 2005 a maio de 2017.

Apesar de muitos estudos destacarem o uso de dados observados com período de 30 anos ou superior (CECÍLIO et al., 2012; PESSOA; CAMPOS et al., 2015; SILVEIRA et al., 2016, SILVA et al., 2017), contudo, utiliza-se a série de dados de 20 anos (1998-2017) em função da carência de estações meteorológicas. Também fez - se uso dos dados observados da estação meteorológica de Óbidos, do Instituto Nacional de Meteorologia, no período de 19802010, para fins de validação.

Normalização dos dados

Com uma abordagem nos padrões temporais da precipitação nos últimos 10 anos, foi adotada a normalização dos dados, ou seja, foi aplicado a Equação 1, para identificar os valores de precipitação no período de menor e maior regime pluviométrico, onde os valores negativos e positivos, referem-se respectivamente a anos com chuva abaixo e acima da média estabelecida (1998-2017).

$$
P_{n}=\frac{P_{i}-\bar{P}}{\sigma}
$$

Onde $P_{n}$ é a precipitação normalizada; $P_{i}$ é a precipitação observada; $\bar{P}$ é a precipitação média e; $\sigma$ é o desvio padrão.

\section{RESULTADOS E DISCUSSÃO}

Observa-se na Figura 2a uma distribuição da precipitação pelo produto CMORPH com uma região predominante de totais anuais superiores a $2.200 \mathrm{~mm}$ nos municípios de Oriximiná e Faro, especialmente na área central da microrregião, com picos de máxima de 
$2.650 \mathrm{~mm}$. Já o município de Óbidos é caracterizado por uma faixa heterogênea em sua precipitação, variando entre $1.500 \mathrm{~mm}$ a $2.400 \mathrm{~mm}$.

As médias de precipitação mensais (Figura 2b) seguem um comportamento descrito por COUTINHO et al. (2018), em que os autores caracterizam a precipitação na bacia do Baixo Amazonas em um período de chuvas e outro de seca. A estação seca ficou concentrada nos meses de junho a novembro com acumulado total de $385 \mathrm{~mm}$. A estação convencional de Óbidos durante o período seco apresentou um quantitativo pluviométrico abaixo dos $100 \mathrm{~mm}$ mensal, o que é consistente com a duração da estação seca na região (GUEDES et al., 2013; SANTOS; OLIVEIRA, 2017).

Observa - se que a concentração de maior acúmulo de precipitação ocorre no mês de março, com um intervalo de transição para o período menos chuvoso, que dar-se-á em maio. Nos meses entre janeiro a abril (período chuvoso) as médias mensais de precipitação mantiveram-se acima dos $250 \mathrm{~mm}$, totalizando um acumulado de $1.385 \mathrm{~mm}$. O mês de agosto, apresentou um quantitativo de chuva crítico, com precipitação mensal média abaixo dos 50 $\mathrm{mm}$.

Figura 2. (a) Distribuição espacial da precipitação média (1998-2017) para a microrregião de Óbidos e (b) temporalidade mensal dos dados observados e estimados para a estação convencional de Óbidos (1980-2010). 

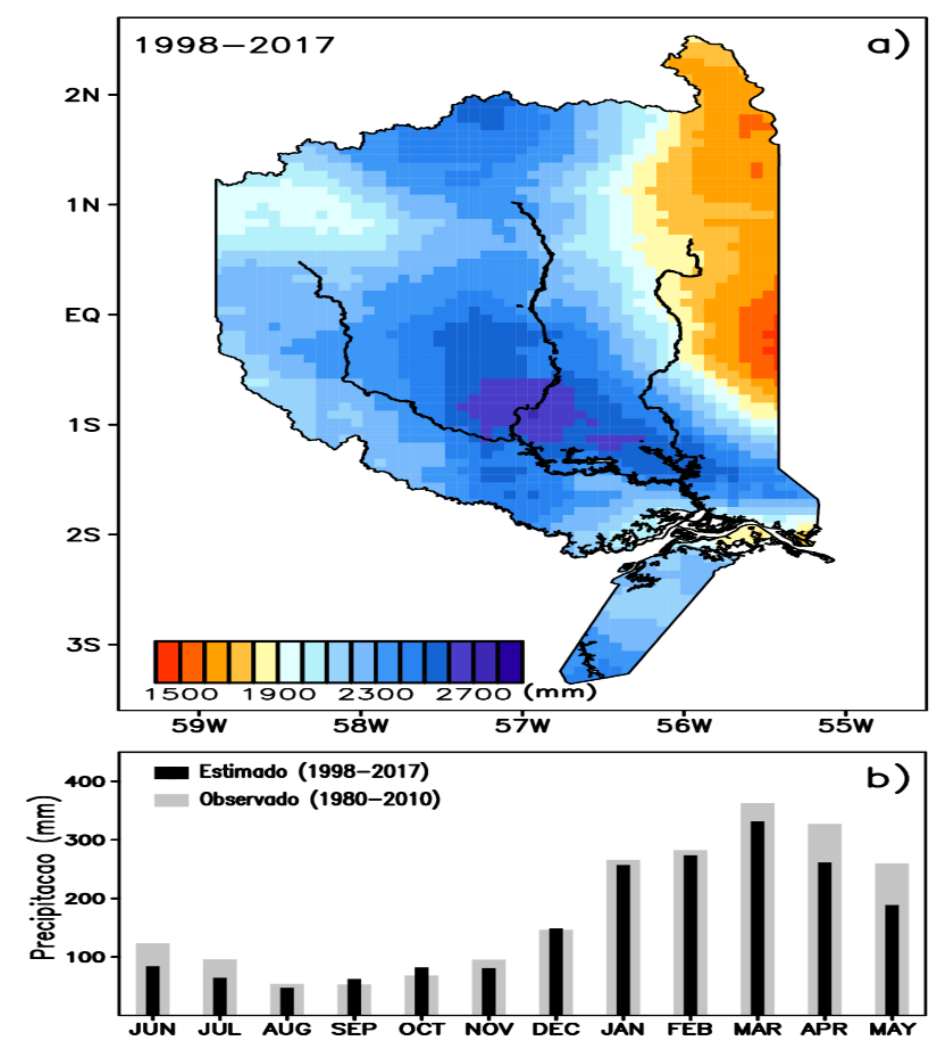

Fonte: Autores (2018)

\section{Análise da variabilidade da precipitação}

A Figura 3 compreende a variabilidade da precipitação nos diferentes anos de ocorrências do ENOS. Observa-se que a variação das chuvas são bastantes expressivas com a atuação de mecanismos como La Niña e El Niño, com seus máximos e mínimos anuais. Esse comparativo das ocorrências do fenômeno ENOS, nas diferentes intensidades (forte, moderado e fraco) com a precipitação, sugere que essas oscilações interanuais das variáveis meteorológicas sobre a região são principalmente devidas às condições climáticas associada aos eventos ENOS e de Dipolo do Atlântico (AMANAJÁS; BRAGA, 2012).

Nota - se, por exemplo, uma forte relação desses eventos com períodos de maior acúmulo pluviométrico (2010-2011), em contraste com os maiores déficits de precipitação (2015-2016) na microrregião de Óbidos, onde destacam - se mecanismos como La Niña (forte) e El Niño (muito forte), respectivamente. Conforme Timpe e Kaplan (2017), uma possível explicação para as proporções hidrológicas de anos afetados por fenômenos ENOS serem maiores (menores) em alguns anos em comparação a outros na Amazônia, deve - se provavelmente ao grau da intensidade destes eventos (Tabela 1). 
Figura 3. Distribuição espacial da precipitação anual estimada entre os anos de 2005 a 2017.
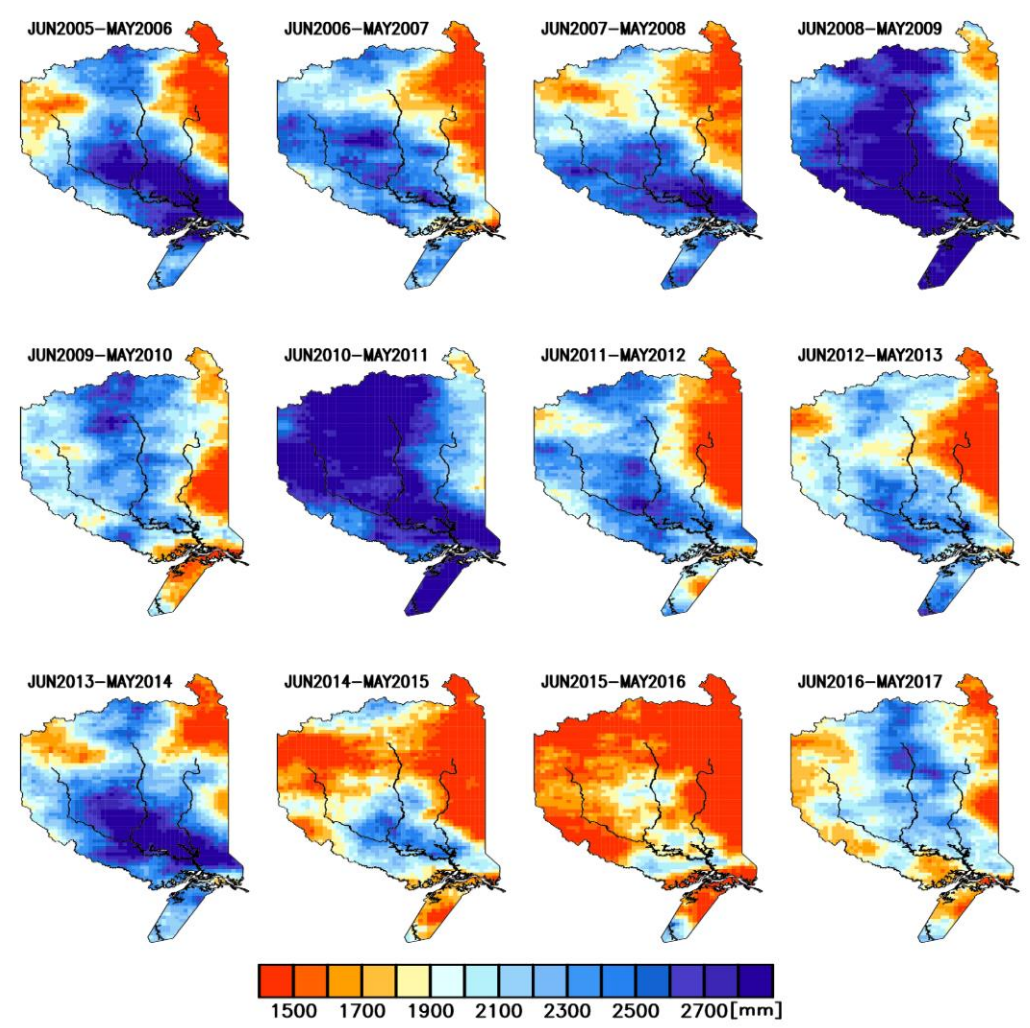

Fonte: Autores (2018).

Tabela 1. Anos de ocorrência de eventos El Niño Oscilação Sul nos últimos 30 anos.

\begin{tabular}{|c|c|c|c|c|c|c|}
\hline \multicolumn{5}{|c|}{ EL Niño } & \multicolumn{3}{c|}{ La Niña } \\
\hline Fraco & Moderado & Forte & Muito Forte & Fraco & Moderado & Forte \\
\hline $2004-2005$ & $1986-1987$ & $1987-1988$ & $1982-1983$ & $2000-2001$ & $1995-996$ & $1988-1989$ \\
$2006-2007$ & $1994-1995$ & $1991-1992$ & $1997-1998$ & $2005-2006$ & $2011-2012$ & $1998-1999$ \\
$2014-2015$ & $2002-2003$ & & $2015-2016$ & $2008-2009$ & & $1999-2000$ \\
& $2009-2010$ & & & $2016-2017$ & & $2007-2008$ \\
& & & & $2017-2018$ & & $2010-2011$ \\
\hline
\end{tabular}

Fonte: Baseado no Oceanic Niño Index (ONI) - NOAA, 2018.

Em análise da Figura 4, constata-se que a precipitação média é superior a 2.340 $\mathrm{mm}$ /ano para os dados observados e $1.879 \mathrm{~mm} /$ ano para os dados estimados, sendo que essa diferença de $461 \mathrm{~mm} /$ ano entre os dados pode ser explicada pela defasagem temporal em 10 anos de observações. Nota - se que existe uma variação de pico de máximas no ano de 20062007 para os dados observados que antagoniza uma ocorrência de El Niño fraco (Tabela 1), sendo que o mesmo não ocorre para os dados estimados, o que pode significar uma maior confiabilidade da variação da precipitação estimada associado aos mecanismos de precipitação (ENOS), já que estudos destacam a possibilidade de falhas nas medições observadas (PENEREIRO et al., 2018). 
A relação dos dados observados e estimados demonstram uma tendência estatisticamente significante $(\mathrm{p} \leq 0.05)$ que se encontra dentro de um intervalo de confiança ao nível de $95 \%$, onde se destaca o desempenho adquirido por dados estimados a partir de sensores de satélite, garantindo uma excelente confiabilidade de aplicações para diversos estudos, cujo a observação in situ é considerada precária ou inexistente sobre a região, representando a variação interanual de precipitação através dos mecanismos de El NiñoOscilação Sul (VAN HUIJGEVOORT et al., 2013; SAURRAL et al., 2016).

Figura 4. Comparativo da precipitação normalizada dos dados observados e estimados da estação meteorológica de Óbidos, entre 2005 e 2017. Barras vermelhas caracterizam as ocorrências para eventos El Niño e barras azuis para eventos de La Niña.

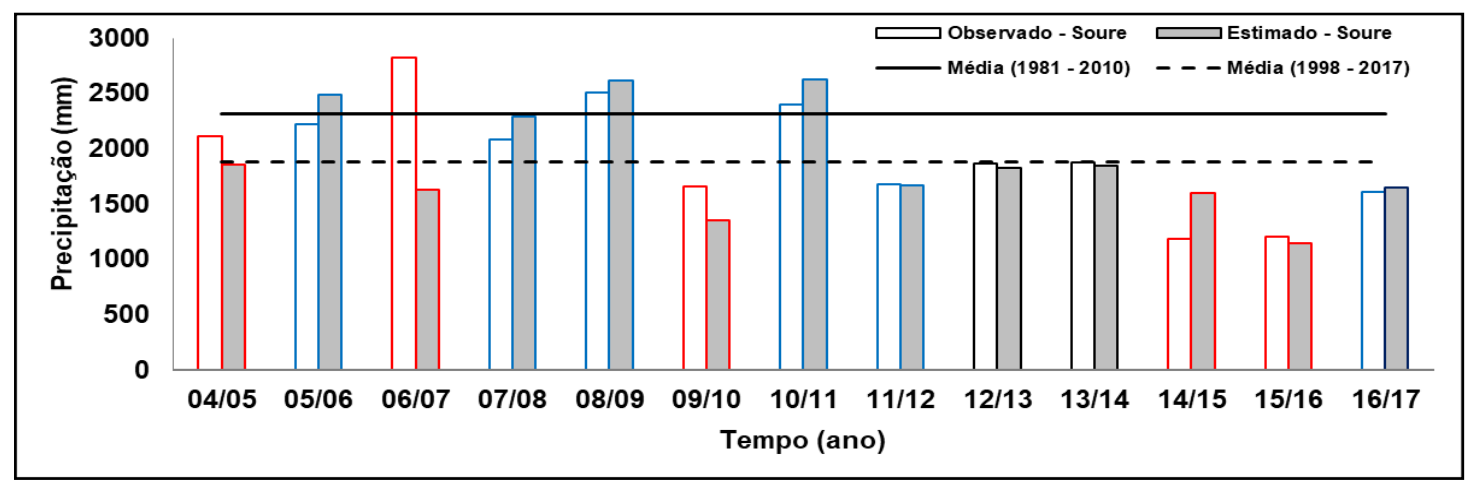

Fonte: Autores (2018).

O resumo dos resultados obtidos para os dados normalizados pode ser observado na Figura 5. Os resultados observados, de modo geral, condizem dos encontrados por COUTINHO et al. (2018). Os autores utilizaram diversas estações pluviométricas (incluindo Óbidos) para a bacia Amazônica, as quais demonstraram que a precipitação normalizada variou, na medida que as ocorrências de ENOS intensificam - se em diferentes mecanismos, como observado em anos de 2008-2009, 2010-2011, 2014-2015 e 2015-2016, coincidindo com os eventos de La Niña (fraca), La Niña (forte), El Niño (fraco) e El Niño (muito forte), respectivamente.

Destaca - se a seca de 2015-2016, com maior severidade, abordada nos estudos de SANTOSO et al. (2017), concordando com a Figura 5 no respectivo ano de análise, que apresenta uma anomalia negativa extremamente elevada, apontando como principal responsável para os problemas relacionados ao déficit hídrico e pluviométrico na microrregião de Óbidos, que provavelmente ocasionou graves implicações para os recursos hídricos, biológicos e nos sistemas socioeconômicos, observados em estudos de outras regiões para a Amazônia (BARNARD et al., 2015; LOPEZ et al., 2017) 
Figura 5. Distribuição espacial da precipitação normalizada entre os anos de 2005 a 2017.
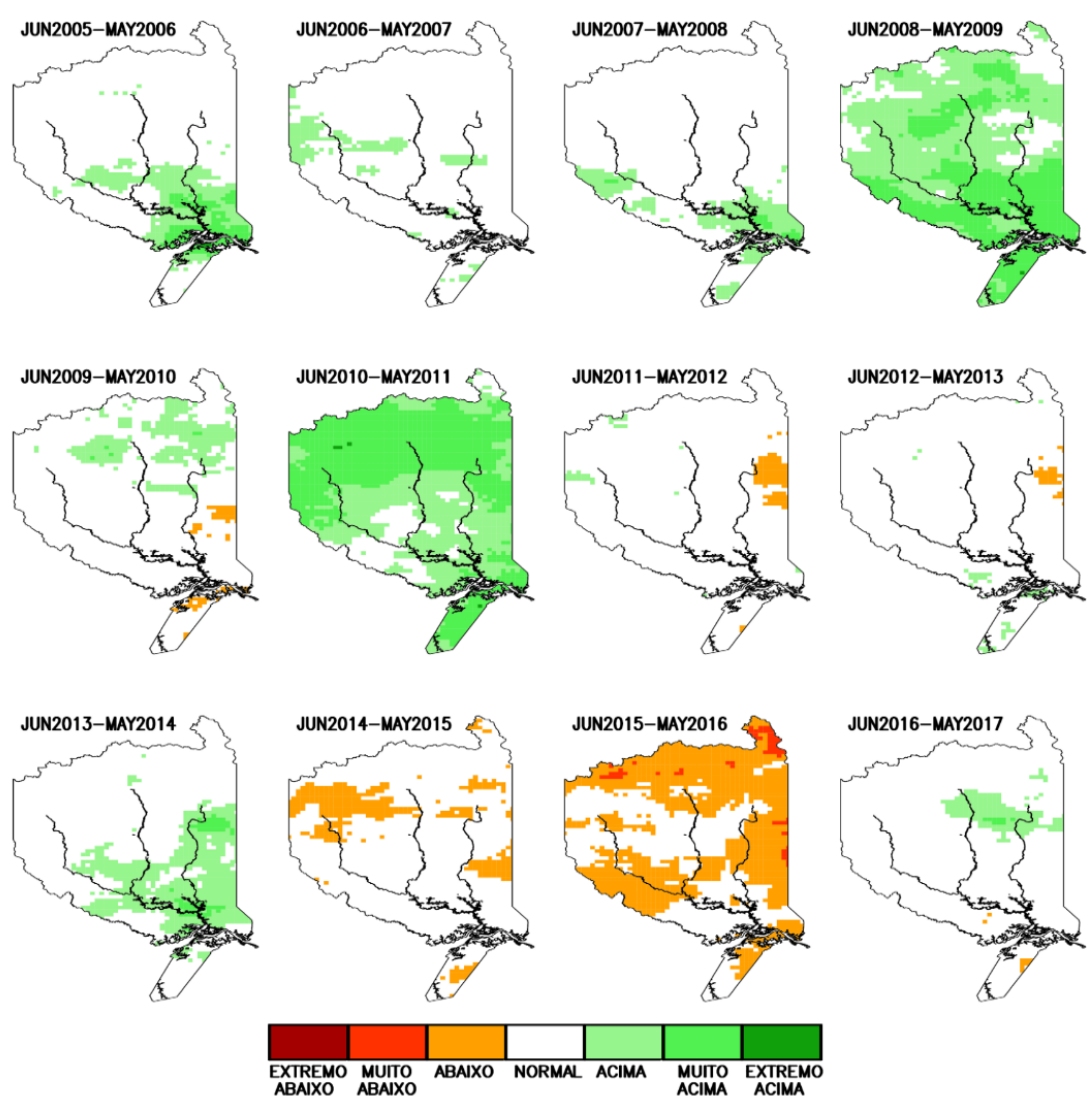

Fonte: Autores (2018).

\section{CONCLUSÕES}

Este artigo apresentou uma visão geral da distribuição geográfica da precipitação na microrregião de Óbidos, fornecendo algumas informações úteis para o desenvolvimento de atividades locais de curto, médio e longo prazo.

Constatou - se a eficácia do produto CMORPH, tanto para áreas com menores acúmulos quanto para maiores acúmulos pluviométricos, sendo corroborado pela validação feita entre os dados medidos e os estimados.

Os dados de precipitação foram satisfatórios para as análises em uma escala interanual, demonstrando sua forte dependência com mecanismos de precipitação, cujo eventos de La Niña e El Niño são responsáveis, em parte, pela modulação nos padrões de chuvas da região, onde uma das principais causas são os efeitos dinâmicos da interação oceano-atmosfera.

\section{AGRADECIMENTOS}


Revista de Geografia (Recife) V. 36, No. 2, 2019 - ISSN: 2238-6211

Agradecemos à Coordenação de Aperfeiçoamento de Pessoal de Nível Superior (Capes) e ao Núcleo de Pesquisa Aplicada ao Desenvolvimento Regional (NUPAD) por toda a assistência na elaboração dos mapas e tratamento dos dados.

\section{REFERÊNCIAS}

ASHOURI, H.; HSU, K-L.; SOROOSHIAN, S.; BRAITHWAITE, D. K.; KNAPP, K. R.; CECIL, L. D.; NELSON, B. R.; PRAT, O. P. PERSIANN-CDR: daily precipitation climate data record from multisatellite observations for hydrological and climate studies. American Meteorological Society, v. 1, n. 1, p. 69-83, 2015.

AMANAJÁS, J. C.; BRAGA, C. C. Padrões espaço-temporal pluviométricos na Amazônia Oriental utilizando análise multivariada. Revista Brasileira de Meteorologia, v. 27, n. 4, p. 423-434, 2012. DOI:

AWANGE, J. L.; FERREIRA, V. G.; FOROOTAN, E.; KHANDU; ANDAM-AKORFUL, S. A.; AGUTU, N. O.; HE, X. F. Uncertainties in remotely sensed precipitation data over Africa. International Journal of Climatology, v. 36, n. 1, p. 303-323, 2016.

BAlSAMO, G., ALBERGEL, C., BELJAARS, A., BOUSSETTA, S., BRUN, E., ClOKE, H.; DEE, D.; DUTRA, E.; MUÑOZ-SABATER, J.; PAPPENBERGER, F.; ROSNAY, P.; STOCKDALE, T.; VITART, F. ERA-Interim/Land: a global land surface reanalysis data set. Hydrology and Earth System Sciences, v. 19, n.1, p. 389-407, 2015.

BARNARD, P. L.; SHORT, A. D.; HARLEY, M. D.; SPLINTER, K. D.; VITOUSEK, S.; TURNER, I. L.; ALLAN, J.; BANNO, M.; BRYAN, K. R.; DORIA, A.; HANSEN, J. E.; KATO, S.; KURIYAMA, Y.; RANDALL-GOODWIN, E.; RUGGIERO, P.; WALKER, I. J.; HEATHFIELD, D. Coastal vulnerability across the Pacific dominated by El Niño-Southern Oscillation. Nature Geoscience, v. 8, n. 1, p. 801-807, 2015. DOI: doi.org/10.1038/ngeo2539

BECK, H. E.; VERGOPOLAN, N.; PAN, M.; LEVIZZANI, V.; VAN-DIJK, A. I. J. M.; WEEDON, G. P.; BROCCA, L.; PAPPENBERGER, F.; HUFFMAN, G. J.; WOOD, E. F. Global-scale evaluation of 22 precipitation datasets using gauge observations and hydrologycal modeling. Hydrological Earth System Science, v. 21, p. 6201-6217, 2017. 
BELAY, S.; ZAITCHIK, B. F.; FOLTZ, J. D. Agroecosystem specific climate vulnerability analysis: application of the livelihood vulnerability index to a tropical highland region. Mitigation and Adaptation Strategies for Global Change, v. 21, n. 1, p. 39-65, 2014.

CECÍlio, R. A.; SILVA, K. R.; XAVIER, A. C.; PEZZOPANE, J. R. M. Método para a espacialização dos elementos do balanço hídrico climatológico. Pesquisa Agropecuária Brasileira, v. 47, n.4, p. 478-488, 2012.

SCHNEIDER, U.; BECKER, A.; FINGER, P.; MEYER-CHRISTOFFER, A.; ZIESE, M.; RUDOLF, B. GPCC's new land surface precipitation climatology based on quality-controlled in situ data and its role in quantifying the global water cycle. Theoretical and Applied Climatology, v. 115, n. 1, p. 15-40, 2014.

COUTINHO, E. C.; ROCHA, E. J. P.; LIMA, A. M. M.; RIBEIRO, H. M. C.; GUTIERREZ, L. A. C. L.; BARbOSA, A. J. S.; PAES, G. K. A. A.; TAVARES, P. A. Variabilidade climática da precipitação na bacia Amazônica brasileira entre 1982 e 2012. Revista Brasileira de Climatologia, v. 22, n. 1, p. 476-500, 2018.

DUAN, Z.; LIU, J.; TUO, Y.; CHIOGNA, G.; DISSE, M. Evaluation of eight high spatial resolution gridded precipitation products in Adige Basin (Italy) at multiple temporal and spatial scales. Science of the Environmental, v. 573, n. 1, p. 1536-1553, 2016.

EMERTON, R.; CLOKE, H. L.; STEPHENS, E. M.; ZSOTER, E.; WOOLNOUGH, S. J.; PAPPENBERGER, F. Complex Picture for likelihood of ENSO-driven flood hazard. Nature Communications, v. 8, n. 1, p. 1-9, 2017.

FALCK, A. S.; VILA, D.; TOMASELlA, J.; MAGGIONI, V.; DINIZ, F. L. R. Avaliação de um modelo estocástico de erro multidimensional aplicado a estimativas de precipitação por satélite. Revista Brasileira de Meteorologia, v. 31, n.1, p. 52-63, 2016.

FAO. Brazilian agriculture: Prospects and challenges. In: OECD-FAO Agricultural Outlook 2015, OECD Publishing, Paris. Disponível em: http://dx.doi.org/10.1787/agr_outlook-2015-5en. Acesso em agos. 2016.

FUNK, C.; PETERSON, P.; LANDSFELD, M.; PEDREROS, D.; VERDIN, J.; SHUKLA, S.; HUSAK, G.; ROWLAND, J.; HARRISON, L.; HOELL, A.; MICHAELSEN, J. The climate 
hazards infrared precipitation with stations - a new environmental record for monitoring extremes. Scientific Data, v. 2, n. 1, p. 1-21, 2015.

GERMANO, M. F.; VITORINO, M. I.; COHEN, J. C. P.; COSTA, G. B.; SOUTO, J. I. O.; REBELO, M. T. C.; SOUSA, A. M. L. Analysis of the breeze circulations in Eastern Amazon: an observational study. Atmosphere Science Letters, n. 18, p. 67-75, 2017.

GUEDES, A. E. D. S.; CÂNDIDO, L. A.; SANTO, A. R. S. R. Variabilidade do estoque de água continental e sua relação com as cheias e vazantes extremas na Amazônia. Revista Ambiente \& Água, v. 8, n. 2, p. 88-99, 2013. DOI: 10.4136/ambi-agua.1137

GUO, H.; CHEN, S.; BAO, A.; HU, J.; Gebregiorgis, A. S.; Xue, X.; Zhang, X. Intercomparison of high-resolution satellite precipitation products over Central Asia. Remote Sensing, n. 7, p. 7181-7211, 2017.

HARRIS, I., JONES, P.D., OSBORN, T.J., LISTER, D.H. Updated high-resolution grids of monthly climatic observations - the CRU TS3.10 Dataset. International Journal of Climatology, v. 34, n. 1, p. 623-642, 2014.

JIMÉNEZ-MUÑOZ, J. C.; MATTAR, C.; BARICHIVICH, J.; SANTAMARÍA-ARTIGAS, A.; TAKAHASHI, K.; MALHI, Y.; SOBRINO, J. A.; SCHRIER, G. Record-breaking warming and extreme drought in the Amazon rainforest during the course of El Niño 20152016. Scientific Reports, v. 6, n. 1, p. 1-7, 2016.

JOYCE, R. J.; JANOWIAK, J. E.; ARKIN, P. A.; XIE, P. CMORPH: A method that produces global precipitation estimates from passive microwave and infrared data at high spatial and temporal resolution. Journal of Hydrometeorology, n. 5, p. 487-503, 2004.

JUNK, W. J.; PIEDADE, M. T. F. The Amazon River basin. In: Fraser, H.; Keddy, A. (Ed.). The world's largest wetlands. Cambridge University Press, 2005, p. 63-117.

KAYANO, M. T.; ANDREOLI, R. V.; SOUZA, R. A. F. Relations between ENSO and South Atlantic SST modes and their effects on the South American rainfall. International Journal of Climatology, v. 33, n. 1, p. 2008-2023, 2013. 
KOSUTH, P.; CALLÈDE, J.; LARAQUE, A.; FILIZOLA, N.; GUYOT, J. L.; SEYLER, P.; FRITSCH, J. M.; GUIMARÃES, V. Sea-tide effects on flows in the lower reaches of the Amazon River. Hydrological Processes, v. 23, n. 22, p. 3141-3150, 2009.

LETTENMAIER, D. P.; ALSDORF, D.; DOZIER, J.; HUFFMAN, G. J.; PAN, M.; WOOD, E. F. Inroads of remote sensing into hydrologic science during the WRR era. Water Resources Research, v. 51, n. 9, p. 7309-7342, 2015.

LIU, J.; DUAN, J.; JIANG, J.; ZHU, A. Evaluation of Three Satellite Precipitation Products TRMM 3B42, CMORPH, and PERSIANN over a Subtropical Watershed in China. Advances in Meteorology, v. 2015, p. 1-13, 2015

LOPEZ, L.; STAHLE, D.; VILlALBA, R.; TORBENSON, M.; FENG, S.; COOK, E. Tree ring reconstructed rainfall over the southern Amazon basin. Geophysical Research Letters, v. 44, n. 14, p. 7410-7418, 2017. DOI: 10.1002/2017g1073363

MARENGO, J. A.; ALVES, L. M.; SOARES, W. R.; RODRIGUEZ, D. A.; CAMARGO, H.; RIVEROS, M. P.; PABLÓ, A. D. Two contrasting severe seasonal extremes in Tropical South America in 2012: flood in Amazonia and drought in Northeast Brazil. Journal of Climate, v. 26, n. 1, p. 9137-9154, 2013.

MARENGO, J. A.; ESPINOZA, J. C. Extreme seasonal droughts and flood in Amazonia: causes, trends and impacts. International Journal of Climatological, v. 36, n. 1, p. 1033$1050,2015$.

MEI, Y. W.; ANAGNOSTOU, E. N.; NIKOLOPOULOS, E. I.; BORGA, M. Error analysis of satellite precipitation products in mountainous basins. Journal of Hydrometeorological, v. 15, n. 1, p. 1778-1793, 2014.

NIKOLOPOUlOS, E. I.; ANAGNOSTOU, E. N.; BORGA, M. Using high-resolution satellite rainfall products to simulate a major flash flood event in northern Italy. Journal of Hydrometeorological, v. 14, n. 1, p. 171-185, 2013.

NOAA, National Oceanic and Atmospheric Administration (2018). Cold and a warn episodes by season. Adaptado de Oceanic Niño Index (ONI). Disponível em: 
www.cpc.ncep.noaa.gov/prodects/analysis_monitoring/ensostuff/ensoyrears.html. Acesso em: maio de 2018.

PENEREIRO, J. C.; BADINGER, A.; MACCHERI, N. A.; MESCHIATTI, M. C. Distribuições de tendências sazonais de temperatura média e precipitação nos biomas brasileiros. Revista Brasileira de Meteorologia, v. 33, n. 1, p. 97-113, 2018. DOI: $10.1590 / 0102-7786331012$

PESSOA, F. E. P.; CAMPOS, J. N. B. Ciclo diário de precipitações pluviais em intervalos de cinco minutos no município de Fortaleza. Revista Brasileira de Meteorologia, v. 30, n.2, p. 195-204, 2015.

ROSA, A. G.; SOUZA, A. M. L.; COSTA, J. A.; SOUZA, E. B. Erosividade da chuva em Rondon do Pará, PA, Brasil de 1999 a 2015 e projetada para 2035. Revista Ambiente \& Água, v. 11, n. 4, p. 1006-2021, 2016.

SANTOS, C. A. C.; OLIVEIRA, V. G. Trends in extreme climate índices for Pará State, Brazil. Revista Brasileira de Meteorologia, v. 32, n. 1, p. 13-24, 2017. DOI: 10.1590/0102778632120150053

SANTOS, M. R. S.; VITORINO, M. I.; PIMENTEL, M. A. S. Vulnerabilidade e mudanças climáticas: análise socioambiental em uma mesorregião da Amazônia. Revista Ambiente \& Água, v. 12, n. 5, p. 842-854, 2017.

SANTOSO, A.; MCPHADEN, M. J.; CAI, W. The defining characteristics of ENSO extremes and the Strong 2015/2016 El Niño. Reviews of Geophysics, v. 55, n. 1, p. 10791129, 2017.

SATYAMURTY, P.; COSTA, C. P. W.; MANZI, A. O. Moisture source for the Amazon Basin: a study of contrasting years. Theoretical and Applied Climatology, v. 111, n. 1, p. 195-209, 2013a.

SATYAMURTY, P.; COSTA, C. P. W.; MANZI, A. O.; CANDIDO, L. A. A quick look at the 2012 record flood in the Amazon basin. Geophysical Research Letters, v. 40, n. 1, p. 1396-1401, 2013 b. 
SAURRAL, R. I.; CAMILONI, I. A.; BARROS, V. R. Low-frequency variability and trends in centennial precipitation stations in southern South America. International Journal of Climatology, v. 37, n. 4, p. 1774-1793, 2016. DOI: 10.1002/joc.4810

SILVA, R. O. B.; MONTENEGRO, S. M. G. L.; SOUZA, W. M. Tendências de mudanças climáticas na precipitação pluviométrica nas bacias hidrográficas do estado de Pernambuco. Engenharia Sanitária Ambiental, v. 22, n. 3, p. 579-589, 2017.

SILVEIRA, C. S.; SOUZA-FILHO, F. A. S.; MARTINS, E. S. P. R.; OLIVEIRA, J. L.; COSTA, A. C.; NOBREGA, M. T.; SOUZA, S. A.; SILVA, R. F. V. Mudanças climáticas na bacia do rio São Francisco: uma análise para precipitação e temperatura. Revista Brasileira de Recursos Hídricos, v. 21, n. 2, p. 416-428, 2016.

SOUZA, A. C.; CANDIDO, L. A.; ANDREOLI, R. V. Variabilidade interanual e fluxo de umidade sobre a Amazônia usando o QTCM. Revista Brasileira de Meteorologia, v. 33, n. 1, p. 41-56, 2018.

SUN, I.; MIAO, C.; DUAN, Q.; ASHOURI, H.; SOROOSHIAN, S.; HSU, K-L. A Review of Global Precipitation Data Sets: Data Sources, Estimation, and Intercomparisons, Reviews of Geophysics, v. 56, n. 1, p. 79-107, 2018.

TAPIADOR, F. J.; TURK, F. J.; PETERSEN, W.; HOU, A. Y.; GARCIA-ORTEGA, E.; MACHADO, L. A. T.; ANGELIS, C. F.; SALIO, P.; KIDD, C.; HUFFMAN, G. J.; CASTRO, M. Global precipitation measurement: methods, datasets and applications. Atmospheric Research, v. 104, n.1, p. 70-97, 2012.

TIMPE, K.; KAPLAN, D. The changing hydrology of dammed Amazon. Science Advances, v. 3 , n. 11, p. 1-13, 2017. DOI: 10.1126/sciadv.1700611

TORRALBA, V.; RODRÍGUEZ-FONSECA, B.; MOHINO, E.; LOSADA, T. The nonstationary influence of the Atlantic and Pacific Niños on North Eastern South American rainfall. Frontiers in Earth Science, v. 3, n. 1, p. 1-10, 2015.

TOTÉ, C.; PATRÍCIO, D.; BOOGAARD, H.; WIJNGAART, V-D.; TARNAVSKY, E.; FUNK, C. Evaluation of satellite estimates for drought and flood monitoring in Mozambique. Remote Sensing, v. 7, p. 1758-1776, 2015. 
TYAQUIÇÃ, P.; VELEDA, D.; LEFÈVRE, N.; ARAÚJO, M.; NORIEGA, C.; CANIAUX, G.; SERVAIN, J.; SILVA, T. Amazon plume salinity response to ocean teleconnections. Frontiers in Marine Science, v. 4, n. 1, p. 1-14, 2017.

VAN HUIJGEVOORT, M. H. J.; HAZENBERG, P.; VAB LANEN, H. A. J.; TEULING, A. J.; ClaRK, D. B.; FOLWELl, S.; GOSLING, S. N.; HANASAKI, N.; HEINKE, J.; KOIRALA, S.; STACKE, T.; VOSS, F.; SHEFFIELD, J.; UIJLENHOET, R. Global multimodel analysis of drought in runoff fro the second half of the twentieth century. Journal of Hydrometeorology, v. 14, p. 1535-1552, 2013. DOI: 10.1175/JHM-D-12-0186.1

WEN, Y.; KIRSTETTER, P.; HONG, Y.; GOURLEY, J. J.; CAO, Q.; ZHANG, J.; FLAMIG, Z.; XUE, X. Evaluation of a method to enhance real-time, ground radar-based rainfall estimates using climatological profiles of reflectivity from space. Journal of Hydrometeorological, v. 17, n. 1, p. 761-775, 2016.

XIE, P. P.; XIONG, A. Y. A conceptual model for constructing high-resolution gaugesatellite merged precipitation analyses. Journal of Geophysical Research Atmospheres, v. 116, n. 21, p. 1-14, 2011.

XIE; P.; JOYCE, R.; WU, S.; YOO, S-H.; YAROSH, Y.; SUN, F.; LIN, R. Reprocessed, Bias-Corrected CMORPH Global High-Resolution Precipitation Estimates from 1998. Journal of Hydrometeorology, v. 18, n. 1, p. 1617-1641, 2017.

ZHAN, W.; PAN, M.; WANDERS, N.; WOOD, E. F. Correction of real-time satellite precipitation with satellite soil moisture observations. Hydrology and Earth System Sciences, v. 19, p. 4275-4291, 2015. 